\title{
Nanotube bundles and tube-tube orientation: A van der Waals Density Functional Study
}

\author{
Heiko Dumlich * and Stephanie Reich \\ Fachbereich Physik, Freie Universität Berlin, 14195 Berlin, Germany
}

(Dated: May 7, 2021)

\begin{abstract}
We study the binding energy, intertube distance and electronic structure of bundles consisting of single walled carbon nanotubes of the same chirality. We model various nanotube structures (chiralities) and orientations with van der Waals density functional theory. The orientation of the tubes in the bundle strongly influences the properties of the bundles if the chirality of the tubes shares symmetry with the trigonal bundle structure, meaning chiralities which have a $60^{\circ}$-rotational symmetry $\left(\mathrm{C}_{6}\right.$-axis), e.g. $(12,0)$ bundles. The bundle structure breaks the symmetry depending on the arrangement of the neighboring tubes. Pseudogaps open in the electronic density of states and intertube distances $( \pm 5-10 \%)$ vary in dependence of the relative orientation of the tubes in the bundle. Bundles of $\mathrm{C}_{6}$-axis armchair tubes have metallic configurations. A $15^{\circ}$ rotation off the high symmetry configuration (AA-stacked) of a $(6,6)$ bundle shows metallic behavior and has higher binding energy than the high symmetry configuration. We find binding energies between $19 \mathrm{meV} /$ atom and $35 \mathrm{meV} /$ atom, depending on the chirality of the tubes. The intertube distances are between $3.2 \AA$ and $3.4 \AA$ but independent of orientation for non $\mathrm{C}_{6}$-axis tubes.
\end{abstract}

PACS numbers: 61.46.-w, 61.48.De, 71.15.Nc, 71.20.-b, 73.22.Gk

\section{INTRODUCTION}

Carbon is one of todays most exciting materials with properties originating from the structure and symme-

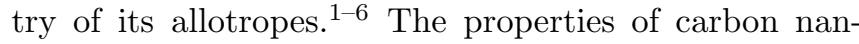
otubes depend on their one dimensionality and the exact arrangement of the carbon atoms on their surface, called chirality $\stackrel{2,4}{*}$ Nanotubes are often found in bundles, ropes or fibers $\stackrel{7}{7}$ These three dimensional structures form through the van der Waals interactions between the tube surfaces, which also influence the properties of the bundles. 8.9 The orientation of tubes in a bundle can be compared to the stacking of graphene layers (e.g. bernal/AB stacked) 10,11 For nanotube bundles, however, we have to additionally consider the chirality of the tubes. The structural influence on the bundle properties is especially interesting for bundles of tubes that consist of only one chirality (monochiral bundles), as these have uniform properties needed for, e.g., electronic devices.

The mechanical and electronic properties of bundles were already studied theoretically and experimentally ${ }^{7,12-17}$ However, there is little knowledge about the influence of the structure (chirality) of the tubes on, e.g., the electronic structure of the bundle. ${ }^{9,10,18}$ Monochiral bundles, have not been experimentally produced yet, even so their production is expected in the near future ${ }^{19-21}$

Nanotube bundles and their electronic structure were studied with density functional theory within the local density approximation (LDA), which allows to consider the structure of the tubes in the bundle $9,10,18$ The local density approximation, however, fails in modeling the van der Waals interaction between tubes. Another approach is to use continuum approximations involving the Lennard-Jones potential. The continuum approximation, however, does not account for the specific configuration of the carbon atoms (structure) ${ }^{22-25}$ Other approaches that consider the structure as well as the van der Waals interaction, do not reach the accuracy of density functional theory calculations $\stackrel{26,27}{2}$ The van der Waals functional developed by Dion et al. was shown to be able to model bundles of carbon nanotubes in density functional theory. ${ }_{28,29}$ At this level of theory studies considering the influence of nanotube chirality in bundles remain missing.

In this paper we study the properties of bundles consisting of carbon nanotubes of the same chirality and handedness by a van der Waals density functional. We calculated the binding energy, intertube distance, and electronic structure in dependence of the orientation of the tubes in the bundle. We find a particularly strong dependence on the orientation of the tubes in the bundle if the tubes are achiral and the chirality of the tubes and the bundle share the trigonal symmetry, meaning that the tubes have a $\mathrm{C}_{6}$-axis. Achiral tubes with $\mathrm{C}_{6}$-axis are metallic, e.g., $(6,6)$ and $(12,0)$ tubes. Pseudogaps open at the Fermi level in dependence of the orientation of the tubes in the bundle. We find metallic behavior for the AA-stacked configuration $\left(0^{\circ}\right)$ for $\mathrm{C}_{6}$-axis armchair bundles, as well as for intermediate configurations which have glide reflection planes. The bundles of $\mathrm{C}_{6}$-axis chiralities experience rotation barriers for the tubes that are induced by the configuration of the atoms on the tube surfaces. The barriers can be as high as $\Delta E=10.5 \mathrm{meV} /$ atom. Rotation barriers for tubes without the trigonal symmetry of the bundle are less than $\Delta E=0.3 \mathrm{meV} /$ atom. The properties of bundles made from non $\mathrm{C}_{6}$-axis tubes have a much weaker dependence on the orientation of the tubes inside of the bundle, e.g. $(8,2)$ and $(14,0)$ tubes.

This paper is organized as follows. We first describe the computational methods used for our study, Sec. II. 
We then discuss the van der Waals energies and intertube distances of bundles in orientational dependence in Sec. III. In Sec. IV we discuss the electronic band structure at the Fermi level for a $(6,6)$ bundle as a function of tube orientation. Section V summarizes this work and discusses the conclusions in a general context.

\section{COMPUTATIONAL METHODS}

We used the ab-initio package SIESTA to perform our density functional theory calculations ${ }^{30}-32$ To optimize the geometry of the isolated tubes we used the generalized gradient approximation parameterized by Perdew, Burke and Ernzerhof, which is good in modeling the interactions within the tube ${ }^{33}$ We calculated the bundle properties (binding strength, intertube distance and bundle electronic structure) with the van der Waals density functional parameterized by Dion et al., which is especially good in modeling the van der Waals interaction between the tubes in the bundle ${ }^{28}$ Both calculations used norm conserving nonlocal pseudopotentials ${ }^{34}$ We balanced the computational time and accuracy, by choosing localized pseudoatomic orbitals with a double$\zeta(\mathrm{DZ})$ basis set to describe the valence electrons. We chose cutoff radii for the $s$ and $p$ orbital of the carbon atoms with $r_{s}=5.949 \mathrm{Bohr}$ and $r_{p}=7.450 \mathrm{Bohr}$. The mesh cutoff for the real-space integration corresponded to about 350 Ry. We used between 10 and $14 k$-points in the Monkhorst-Pack scheme ${ }^{35}$ to calculate the total energies in the $k_{z}$-direction. The $z$-axis was chosen as the tube-axis. We performed all total energy calculations with only one $k$ point in the $x$ and $y$ direction. The $k$ point sampling for the band structure calculations of the bundled tubes were $20 k$-points in $x$ and $y$ direction leading to a $k$ point sampling of $20 \times 20 \times 300 k$-points for $(n, 0)$ zigzag tubes, $20 \times 20 \times 300$ for $(n, n)$ armchair tubes, and $20 \times 20 \times 200$ for the chiral $(8,2)$ tube. In this paper we will only consider the band structure of the monochiral $(6,6)$ armchair tube bundle in detail.

To obtain decent bundle structures we first optimized the lattice constant of isolated tubes by minimization of the total energy in dependence of the lattice constant. We then performed a geometry optimization for isolated carbon nanotubes within the generalized gradient approximation to derive an initial tube geometry for our bundled tube calculations. We used the conjugate gradient method to optimize the atomic coordinates to a maximal force tolerance of $0.04 \mathrm{eV} / \AA$, which is adequate for our accuracy. The optimized isolated tube coordinates were then used to perform the van der Waals density functional calculations.

For the van der Waals density functional calculations we placed a tube in a hexagonal unit cell with periodic boundaries, see Fig. 1 for the example of a bundle of $(6,6)$-tubes. We calculate a bundle by an infinite number of tubes (bulk-bundle), which serves as a model of an inner tube $I$ in a real carbon nanotube bundle.

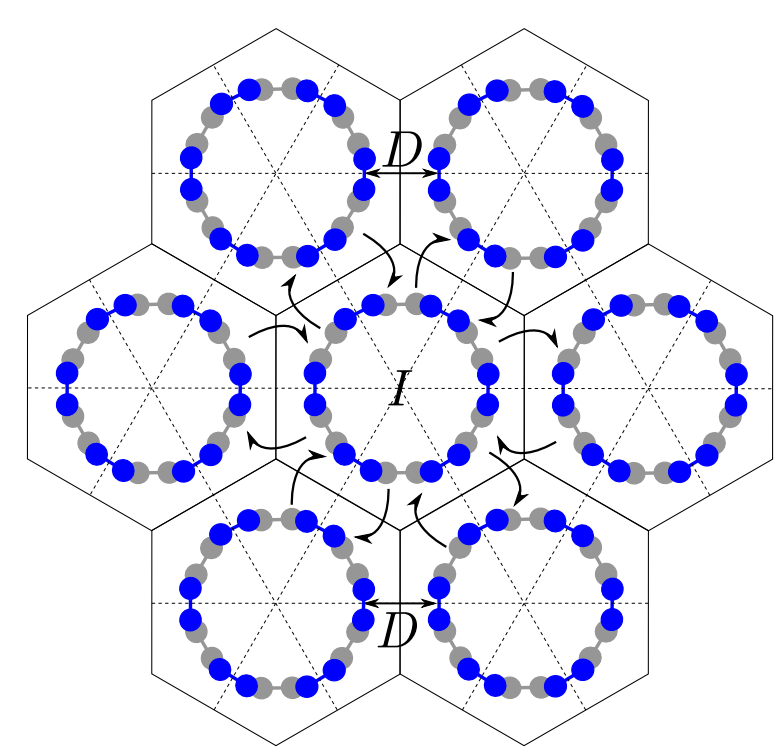

FIG. 1: Ball and stick sketch of a bundle of seven $(6,6)$-tubes in their high-symmetry orientation $\left(0^{\circ}\right)$. The hexagon in the middle (inner tube $I$ ) represents the unit cell for our bundle calculations. Lines connecting the middle points of the hexagons were added for clarity. The top carbon atoms are highlighted in blue, bottom carbon atoms are gray. The symmetry for seven tubes is trigonal. The inner tube $I$ interacts with six neighbors with intertube distance $D$.

The total energy of the bulk bundle was minimized in dependence of the intertube distance to obtain the optimal distance between the tubes of the bundle as presented in Fig. 2, A Lennard-Jones potential is plotted with $\epsilon$ and $r_{\min }$ parameters fitted to our data. The Lennard-Jones potential shows a smaller width in the attractive region compared to the calculated data points. We did not perform a structure relaxation with the van der Waals density functional as this leads to energetically lowest states, which does not allow to consider energetically unstable orientations of the tubes of the bundle. Therefore, stress remains in the bundle systems depending on the chirality and to a smaller extent on the orientation of the tubes. The maximal forces in the systems are $F_{\text {max }} \approx 0.3 \mathrm{eV} / \AA$ for armchair bundles, $F_{\max } \approx 1 \mathrm{eV} / \AA$ for zigzag bundles, and $F_{\max } \approx 3 \mathrm{eV} / \AA$ for the $(8,2)$ and $(12,6)$ bundle. Previous studies of tube-tube interaction found the distortion caused by the bundling to have little or no effect on the properties for tubes with diameters below $15 \AA .9 .36$

The total van der Waals energy is defined as the difference between minimal total energy of the bundle and total energy of the isolated tube (both calculated with the functional of Dion et al. $\stackrel{28}{=}$ ), which corresponds to infinite intertube distance. Dividing through the number of atoms of the unit cell yields the van der Waals energy per atom. We derive the intertube distance from the optimized $x y$-unit cell length by subtraction of the diameter of the tube. The band structure was plotted for certain high symmetry directions. We used the whole $k$-sampled 


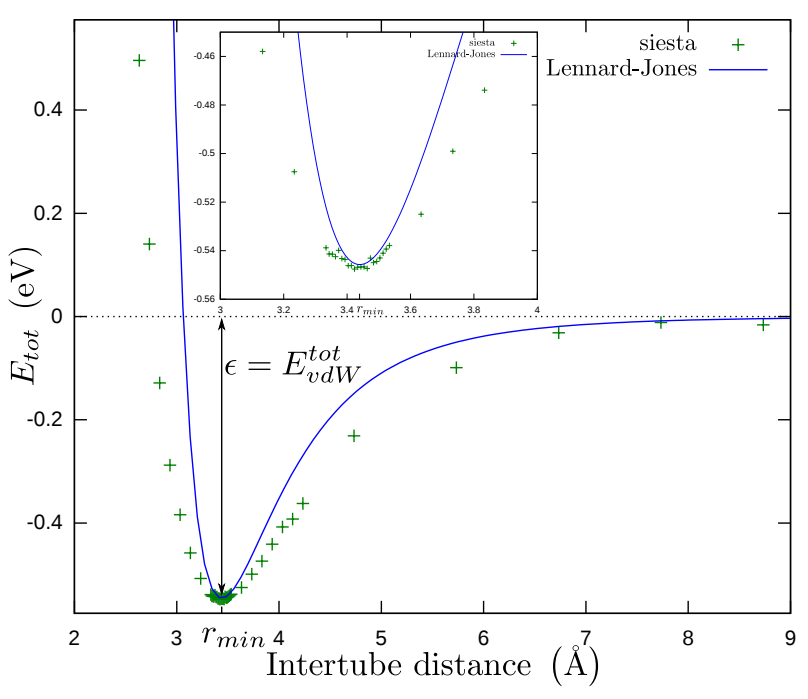

FIG. 2: Total energy in dependence of the intertube distance calculated for a bundle of $(6,6)$ tubes. The energy is normalized to the energy of an isolated tube, which corresponds to infinitely distant neigboring tubes. The data points were obtained by SIESTA. A Lennard-Jones potential is plotted with $\epsilon$ and $r_{\text {min }}$ parameters fitted to our data.

zone to derive the density of states at an electronic temperature of $20 \mathrm{~K}$.

\section{VAN DER WAALS ENERGIES AND INTERTUBE DISTANCES}

We start this section with a discussion of the symmetry properties of nanotube bundles. This discussion is followed by a study of the influence of the orientation of tubes in the bundle on the van der Waals energies between the tubes of the bundle, as well as the energetically optimized intertube distances. At the end of the section we draw a conclusion from our results for the properties of mixed chirality bundles and monochiral bundles.

Bundles were experimentally observed in a triangular lattice that contained up to hundreds of tubes $\stackrel{7.37}{\underline{I n} \text { our }}$ calculations we model the triangular lattice of the bundle by a hexagonal unit cell, compare Fig. 1. A symmetry breaking occurs, if the individual tube and the bundle do not share all symmetry operations. The bundle structure has in general a $\mathrm{D}_{6 h}$ symmetry, this means it has symmetry axes $\left(2 \mathrm{C}_{6}, 2 \mathrm{C}_{3}, \mathrm{C}_{2}, 3 \mathrm{C}_{2}, 3 \mathrm{C}_{2}{ }_{2}\right)$, mirror planes (3 $\left.\sigma_{v}, 3 \sigma_{d}, \sigma_{h}\right)$, one inversion center $(i)$, rotation-reflection axes $\left(2 \mathrm{~S}_{3}, 2 \mathrm{~S}_{6}\right)$ and one identity element $(E)$. The tubes have symmetry operations depending on their chirality. The level of symmetry breaking depends on the number of shared symmetry elements between the bundle structure and the chirality of the individual tube. A tube in the bundle has to have the same atom configuration every $60^{\circ}$ on the circumference of the tube to share symmetry operations with the bundle. If the chirality and bundle structure share the symmetry, we say that the chirality
TABLE I: Comparison of the intertube distance $D$, van der Waals energy per atom $\left|E_{v d W}^{a t o m}\right|$, and their variations $\Delta\left(D, E_{v d W}^{a t o m}\right)$ for various chiralities $(n, m)$, chiral angles $\theta$ and tube diameters $d$. The table presents the dependence of the fundamental properties on the orientation (rotation) of the tubes in the bundle with minimal and maximal values. Errors for intertube distances are $\pm 0.02 \AA$ and for binding energies the errors are $\pm 0.2 \mathrm{meV} /$ atom.

\begin{tabular}{ccccccc}
$(n, m)$ & $\theta\left(^{\circ}\right)$ & $d(\AA)$ & $D(\AA)$ & $\Delta D$ & $\left|E_{v d W}^{\text {atom }}\right|(\mathrm{meV})$ & $\Delta E_{v d W}^{\text {atom }}$ \\
\hline$(8,0)$ & 0 & 6.41 & $3.29-3.32$ & 0.03 & $31.1-31.3$ & 0.2 \\
$(5,5)$ & 30 & 6.92 & $3.33-3.35$ & 0.02 & $29.5-29.6$ & 0.1 \\
$(9,0)$ & 0 & 7.19 & $3.17-3.35$ & 0.18 & $29.5-34.7$ & 5.2 \\
$(8,2)$ & 10.9 & 7.31 & $3.29-3.32$ & 0.03 & $29.3-29.6$ & 0.3 \\
$(6,6)$ & 30 & 8.27 & $3.15-3.44$ & 0.29 & $22.7-33.2$ & 10.5 \\
$(12,0)$ & 0 & 9.53 & $3.25-3.40$ & 0.15 & $23.9-26.9$ & 3.0 \\
$(14,0)$ & 0 & 11.10 & $3.31-3.33$ & 0.02 & $23.8-24.0$ & 0.2 \\
$(12,6)$ & 19.1 & 12.56 & $3.26-3.30$ & 0.04 & $21.6-22.5$ & 0.9 \\
$(10,10)$ & 30 & 13.70 & $3.27-3.31$ & 0.04 & $22.2-22.5$ & 0.3 \\
$(12,12)$ & 30 & 16.42 & $3.22-3.37$ & 0.15 & $18.8-21.5$ & 2.7 \\
\hline
\end{tabular}

has a $\mathrm{C}_{6}$-axis and symmetry breaking is lifted in high symmetry configurations (e.g. $0^{\circ}$ in Fig. 1). The high symmetry configuration has all symmetry operations of the $\mathrm{D}_{6 h}$ symmetry, especially mirror planes.

We study the orientational influence on the properties of the bundles by simultaneous rotation of all tubes of the bundle in steps of $1^{\circ}$ or $5^{\circ}$ starting from $0^{\circ}$ to $60^{\circ}$. We consider the $(6,6),(12,12),(9,0),(12,0)$ and $(12,6)$ $\mathrm{C}_{6}$-axis chiralities. The $(12,0)$-tube structure, for example, has the same atomic configuration every $15^{\circ}$ around the circumference (considering screw operations), as the full circumference contains $360^{\circ}$ and there are 24 atom positions on the circumference of the tube. This also means that the $(12,0)$-tube has the same atomic configuration at the angles $0^{\circ} / 360^{\circ}, 60^{\circ}, 120^{\circ}, 180^{\circ}, 240^{\circ}$ and $300^{\circ}$, therefore it has a $\mathrm{C}_{6}$-axis. To study the effect of intertube orientation on non $\mathrm{C}_{6}$-axis chiralities we further study the chiral $(8,2)$-bundle, the two zigzag bundles $(8,0)$ and $(14,0)$ and the two armchair bundles $(5,5)$ and $(10,10)$, which have nearly no common symmetry elements with the bundle structure.

We present the minimal values, maximal values and variation in dependence of the orientation of the tubes (rotation angle) for the intertube distance $\mathrm{D}$ and van der Waals energy per atom $\left|E_{v d W}^{a t o m}\right|$ for various chiralities and tube diameters, see Tab. [I. We only see small variations for the values of non $\mathrm{C}_{6}$-axis chiralities, e.g. for the bundle of $(8,2)$-tubes. The intertube distance of the $(8,2)$-bundle varies by $\Delta D \approx 0.03 \AA$ and $E_{v d W}^{a t o m}$ varies by $\approx 0.3 \mathrm{meV}$, meaning by $1 \%$ or less. Our results correspond well to results of the $(10,10)$-bundle, with our maximal difference between lowest and highest van der Waals energy per atom $\Delta E_{v d W}=0.3 \mathrm{meV}$ and the activation barrier for rotations of $\Delta E_{v d W}=0.15 \mathrm{meV}$ reported previously $\underline{14}$ The activation barrier results from the reduction in symmetry from $\mathrm{D}_{2 h}$ to $\mathrm{C}_{2 h}$ due to rota- 
tion 15 The properties of bundles made from non $\mathrm{C}_{6}$-axis chiralities show hardly any dependence on orientation.

Comparing the intertube distance for the $(8,0)$ and the $(14,0)$ tube, we find a variation of less than $1-2 \%$, whereas the tube diameter increases by $\approx 73 \%$. The van der Waals energy per atom decreases about $23 \%$ for the same diameter comparison. The tube diameter has a strong influence on the binding strength, but does not influence the intertube distance.

For the $\mathrm{C}_{6}$-axis chiralities, we receive a radically different result for the dependence of the properties of the bundles on the rotation angle. We observe a variation of the bundle properties in dependence of the orientation for all $\mathrm{C}_{6}$-axis chiralities, compare Tab. I Starting with the zigzag chirality $(9,0)$; The intertube distance varies by $\Delta D \approx 0.18 \AA$, corresponding to about $6 \%$ variation, which is six times higher than the variation observed for non $\mathrm{C}_{6}$-axis chiralities and at least three times higher than the variation associated with tube diameter. Furthermore $\Delta E_{v d W}^{a t o m} \approx 5.2 \mathrm{meV}$, corresponding to about $15 \%$ variation, which is 15 times higher than the variation observed for non $\mathrm{C}_{6}$-axis chiralities and only slightly smaller than the variation accounted to the tube diameter. For the $(12,0)$ chirality the orientational dependence on the properties weakens, with $\Delta D \approx 0.15 \AA$ $(\approx 4 \%)$ and $\Delta E_{v d W}^{a t o m} \approx 3 \mathrm{meV}(\approx 11 \%)$. The rotation energy barrier $\Delta E_{v d W}^{a t o m} \approx 3.0 \mathrm{meV}$ corresponds well to the barrier of $3.0 \mathrm{meV}$ previously reported by LDA calculations. 10 The influence of the orientation on the bundle properties decreases with increasing tube diameter limiting the occurrence of the special properties of the $\mathrm{C}_{6}$-axis bundles to small diameter nanotubes. We suppose that the increase in interaction area lowers the influence of the local symmetry. The interaction area increases through the reduced curvature of larger-diameter tubes.

The $(6,6)$ armchair bundle shows the strongest orientation dependence with $\Delta D \approx 0.29 \AA(\approx 9 \%)$. The rotation energy barrier $\Delta E_{v d W}^{\text {atom }} \approx 10.5 \mathrm{meV}(\approx 32 \%)$ is larger than $\approx 5.0 \mathrm{meV}$ previously reported by LDA calculations. ${ }^{10}$ Interestingly, the variations of the bundle parameters in dependence of the orientation are smaller for the zigzag bundles than for the armchair bundles of comparable tube diameter.

For $\mathrm{C}_{6}$-axis bundles of chiral tubes (e.g. $\left.(12,6)\right)$ we see a dependence on the van der Waals energy $\left(\Delta E_{v d W}^{a t o m} \approx\right.$ $0.9 \mathrm{meV}(\approx 4 \%))$ and the intertube distance $(\Delta D \approx$ $0.04 \AA(\approx 1 \%))$, however, the dependence is low. In chiral $\mathrm{C}_{6}$-axis bundles alignment of neighboring tubes is impossible due to the handedness of chiral tubes. Neighboring tubes with same handedness have opposing surfaces with different handedness leading to mismatched surface structures of neighboring surface atom layers. It is not possible to interchange handedness of neighboring tubes to generate alignment for all neighbors as neighbors with same handedness remain caused through the trigonal structure of the bundle.

The intertube distance of achiral $\mathrm{C}_{6}$-axis nanotubes depends on their orientation and varies by as much as

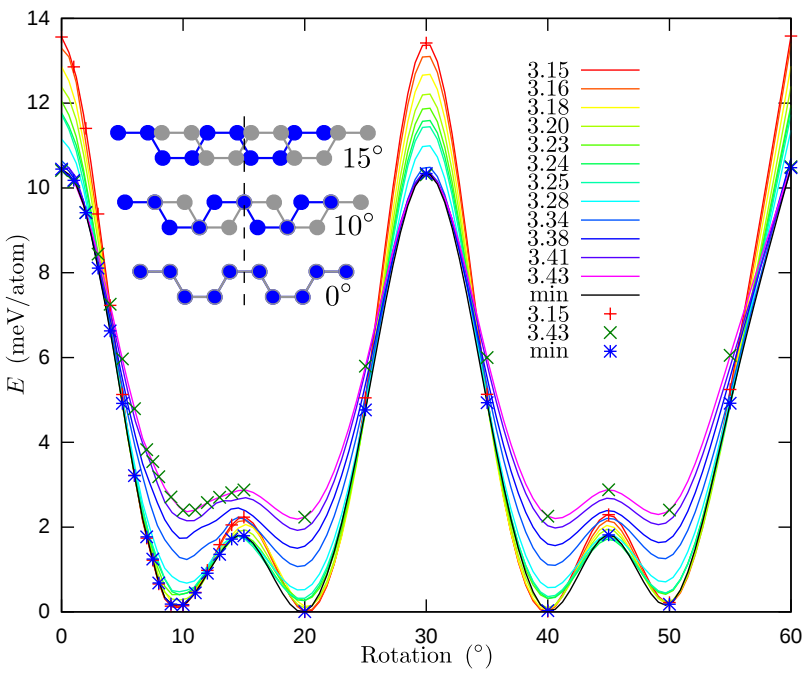

FIG. 3: Total energy for various intertube distances as a function of rotation angle for a bundle of $(6,6)$-tubes. The total energy depends on the intertube distance as well as the orientation of the tubes. The inset shows a side view sketch of the atomic configuration for the three most interesting rotation angles. The front tube has blue atoms, the tube in the back is in gray.

$10 \%$. The binding strength of $\mathrm{C}_{6}$-axis bundles is influenced through the variation of the intertube distance but at the same time by the orientation; see Fig. 3. The influence of the intertube distance on the total energy has a strong impact for $0^{\circ}$ (AA-stacked) and around $10^{\circ}$ (ABstacked), but is weaker around $4^{\circ}$. The orientational influence on the binding strength of $\mathrm{C}_{6}$-axis chiralities with small diameters $(<20 \AA)$ is of the same magnitude as the influence of the tube diameter on the binding strength in the bundle.

We use the example of the $(6,6)$-bundle to discuss the physical properties of a $\mathrm{C}_{6}$-axis bundle. In this bundle the properties depend most strongly on rotational orientation. The van der Waals energy per atom of the $(6,6)$ bundle as a function of rotation angle shows symmetry breaking behavior, see Fig. 4. Symmetry is initially $\mathrm{D}_{6 h}$ $\left(0^{\circ}\right)$ and reduces to $\mathrm{C}_{6 h}$ (loss of mirror planes) due to rotation. The high symmetry configuration of $0^{\circ}$, corresponding to AA stacking in graphite ${ }^{10}$, is not energetically stable, see Fig. 3. It has one of the highest intertube distances of $3.43 \AA$. The magnitude of the van der Waals energy increases up to a maximum at $\approx 10^{\circ}$, corresponding to $\mathrm{AB}$ stacking in graphite ${ }^{10}$, see Fig. 4. This is in contrast to previous LDA findings, where a configuration about $2.5^{\circ}$ off the $\mathrm{AB}$ stacking lead to a maximum at about $7.5^{\circ}, 10$ The configuration at $10^{\circ}$ has one of the smallest intertube distances observed in our calculations with $3.15 \AA$. At $15^{\circ}$ the rotation leads to an interesting configuration that has glide reflection planes, with a local binding energy minimum and a moderate intertube distance of $3.25 \AA$. Further rotations only reproduce the behavior that is contained in the first $15^{\circ}$ rotation, see 


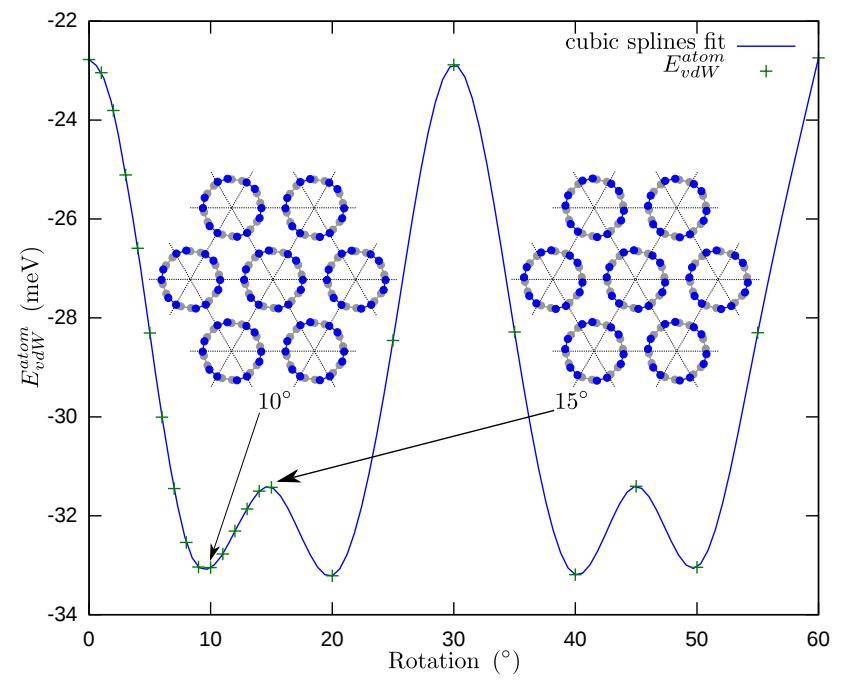

FIG. 4: Van der Waals energy per atom $E_{v d W}^{a t o m}$ as a function of rotation angle for a bundle of $(6,6)$-tubes. The tubes of the bundle are rotated starting from the high symmetry position $\left(0^{\circ}\right.$, see Fig. 11). The lines are cubic spline fits and show symmetry breaking behavior. Global extrema occur every $10^{\circ}$. Minimum values occur at $10^{\circ}+30^{\circ} \cdot n$ and $20^{\circ}+30^{\circ} \cdot n$ with integer $n$. Global maxima occur every $30^{\circ}$ starting from $0^{\circ}$ and local maxima occur every $30^{\circ}$ starting from $15^{\circ}$. The insets show the two exemplary configurations of $10^{\circ}$ and $15^{\circ}$ rotation of the tubes in the bundle. The blue spheres mark the position of the top carbon atoms, the gray spheres mark the position of the bottom carbon atoms.

\section{Fig. 4}

The error in our calculation can be estimated by comparing two identical configurations, e.g. $10^{\circ}, 20^{\circ}, 40^{\circ}$, and $50^{\circ}$ in Fig. 4. We obtain an error of $\pm 0.2 \mathrm{meV} /$ atom for the total energy. An error of $\pm 0.02 \AA$ for the intertube distance can be estimated from the data in Fig. 3 ,

The smaller activation barrier for rotations between $15^{\circ}$ and $20^{\circ}$ has a value of $\Delta E=1.8 \mathrm{meV} /$ atom and the second, larger activation barrier for rotations between all orientations (e.g. between $0^{\circ}$ and $10^{\circ}$ ) is $\Delta E=10.5 \mathrm{meV} /$ atom. Rotations of a solid made from $C_{60}$ fullerenes were experimentally and theoretically found to be hindered below $T \approx 260 \mathrm{~K}$, implicating that a hinderance of rotations for nanotubes in a bundle might also be possible ${ }^{14,38-40}$

At the end of this section we want to discuss our findings and the implications for mixed chirality carbon nanotube bundles. Most carbon nanotube bundles contain nanotubes of various chiralities $(n, m)$. The binding strength and intertube distance depend in general on the orientation of the tubes in the bundle. But the orientational dependence of the aforementioned properties is suppressed by symmetry breaking induced by mismatch of the bundle and tube symmetry. We can conclude from our calculations, that the properties of bundles of mixed chiralities have a negligible dependence on tube orientation. This results from the non $\mathrm{C}_{6}$-axis chirality observations, which show only a weak dependence on the

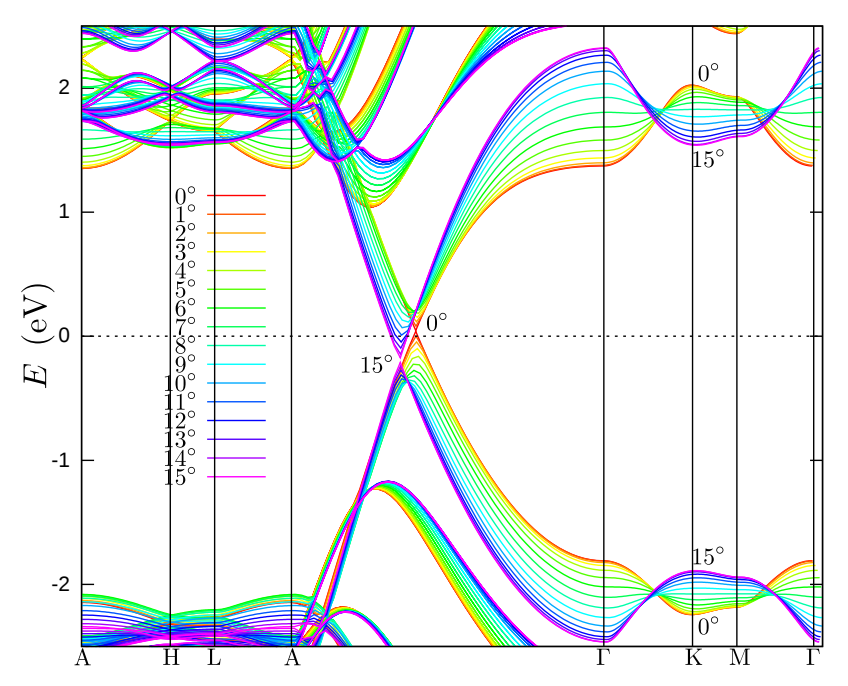

FIG. 5: Band structure along high symmetry directions of a bundle of $(6,6)$-tubes rotated starting from its high symmetry configuration $\left(0^{\circ}\right)$ in rainbow colors (color online). The band structure diagrams are normalized to the Fermi levels.

orientation for the bundle properties (about or less than $1 \%$ variation in orientation dependence). The tube diameter (curvature) otherwise has a strong influence on the binding strength, but does not influence the intertube distance. Recently Crochet et al. succeeded in producing nearly mono chirality single walled carbon nanotube bundles, highlighting the possibility to produce monochiral samples in the near future which would allow to experimentally test the theoretically predicted properties. ${ }^{19-21}$ Only the monochiral $\mathrm{C}_{6}$-axis tubes preserve the symmetry in the higher symmetry configurations. Chiralities with $\mathrm{C}_{6}$-axis can exhibit special properties, e.g. binding energy and intertube distance are influenced in orientational dependence in contrast to the tube diameter which only influences the binding energy.

\section{ELECTRONIC BAND STRUCTURE AT THE FERMI LEVEL}

In this section we study the band structure, density of states, and pseudogap and their variation in bundles of metallic tubes belonging to the $\mathrm{C}_{6}$-axis chirality at the example of $(6,6)$-tubes. At the end of the section we compare our results to other theoretical and experimental studies and discuss the influence of rotation barriers on the electronic properties of bundles.

Band structure diagrams of a bundle of $(6,6)$-tubes for orientations of $0^{\circ}$ to $15^{\circ}$ show that the bands change their slope with orientation, see Fig. 5. The valence band maximum and conduction band minimum shift in energyand $k$-direction through the change in orientation. This is accompanied by a shift of the Fermi level, as can be seen between $\mathrm{A}$ and $\Gamma$ (all Fermi levels were normalized to $0 \mathrm{eV}$ ). The Fermi levels shift through the changed 


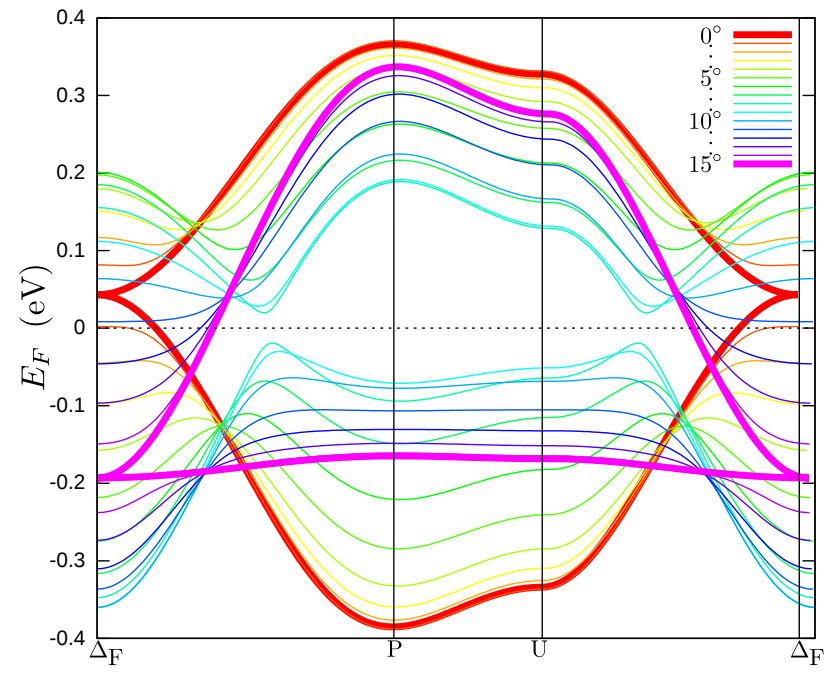

FIG. 6: Band structure along high symmetry directions of a bundle of $(6,6)$-tubes starting from the position of the valence and conduction band crossing in rainbow colors (color online). The band structure diagrams are normalized to the Fermi levels. The valence band flattens by change in orientation while the influence on the conduction band is smaller and it mostly keeps its shape. The bands for the orientations with metallic behavior (without pseudogap) are highlighted.

electron density of each orientation, which result from a volume change induced by the orientational specific intertube distances. For the high symmetry configuration $\left(0^{\circ}, \mathrm{D}_{6 h}\right)$ the valence band and conduction band cross at about $43 \mathrm{meV}$ above the Fermi level, which corresponds well to the value of about $70 \mathrm{meV}$ calculated within LDA calculations; the Fermi level is shifted compared to the Fermi level of the isolated tube.$^{\frac{9}{}}$ The $k_{z}$ value for the position of the valence band maximum and conduction band minimum is between $k_{z}=0.60 \cdot \frac{\pi}{a}$ for $0^{\circ}$ and $k_{z}=0.66 \cdot \frac{\pi}{a}$ for $15^{\circ}$, where $a=2.491 \AA$ is the lattice constant along the tube. Our $k_{z}$-values correspond well to the value in isolated tubes of $k_{z}=2 / 3 \cdot \frac{\pi}{a}$. There is no band splitting in the orientations at $0^{\circ}$ and $15^{\circ}$. The different parity of the bands allows the crossing of the bands. The band splits by rotating the tubes of the bundle as little as $1^{\circ}$ out of the high symmetry position, which opens up a band gap of $E_{g}=75 \mathrm{meV}$ along the $\mathrm{A} \Gamma$-direction. The mirror symmetry for rotated configurations is broken, which leads to anticrossing. The band gap increases until $\approx 7^{\circ}$ and then becomes smaller again until it vanishes at $15^{\circ}$. The valence band flattens in dependence of the orientation for the high symmetry directions starting from the valence and conduction band crossing, see Fig. 6 .

The high symmetry configuration $\left(0^{\circ}\right)$ shows metallic behavior while an increase of rotation angle of the tubes in the bundle increases a pseudogap, which is maximal at $\approx 7^{\circ}$, see density of states in Fig. 7. The pseudogap diminishes with further rotation until it closes at $15^{\circ}$, which can be accounted for by the symmetry of the con- figuration. The $15^{\circ}$ configuration looses mirror planes, but has glide reflection planes, which keeps the configuration metallic, see Figs. 6 and 7, Furthermore, the density of states minima shift around the Fermi level from $0 \mathrm{meV}$ at the global minimum to $50 \mathrm{meV}$ in dependence of the orientation, see right panel in Fig. 7 . The shift of the density of states maxima leads to increased density of states at the Fermi level for orientations between $12^{\circ}$ and $14^{\circ}$, compared to the metal like behavior of the $0^{\circ}$ orientation. This can partly be understood by comparison to the behavior observed by the band structure, see Fig. 5 .

The orientation has a smaller influence on the valence side than on the conduction side of the band structure, see Fig. 7. The first peak of the conduction side shifts between $1.07 \mathrm{eV}$ and $1.22 \mathrm{eV}$ through rotation; for certain rotation angles (e.g. $15^{\circ}$ ) it becomes a shoulder. The valence band shows only small variation for the first two peaks.

We conclude that the most exciting properties of $\mathrm{C}_{6}$ axis bundles can be found in the band structure and density of states. Achiral $\mathrm{C}_{6}$-axis bundles can be metallic. Certain orientations possess higher density of states at the Fermi level than the metallic configurations. The minima of the density of states meanwhile shift around the Fermi level in dependence of the orientation. The band structure shows a dive of the conduction band minimum below the Fermi level along the high symmetry direction accompanied by a pseudogap opening which closes again for higher symmetry configurations that have mirror planes or glide reflection planes.

We now want to compare our results to other theoretical studies. The density of states of the high symmetry and rotated $(10,10)$ monochiral nanotube bundles were shown to have very similar behavior, which agrees with our results $\frac{14,15}{}$ For $\mathrm{C}_{6}$-axis bundles, however, we find a strong coupling between the electronic states near the Fermi level and the rotational motion, which was suggested to lead to superconducting behavior previously 14 In contrast to previous studies we find metallic behavior for achiral $\mathrm{C}_{6}$-axis bundles (e.g. $\left.(6,6),(12,12)\right)$ for multiple orientations. 10 We were able to show, that no energy gap, but a pseudogap opens in dependence of orientation of the tubes in the bundle in contrast to previous studies, which showed an energy gap in the density of states for AB-stacked $(6,6)$ bundles as well as for the $8^{\circ}$ orientation 10 Furthermore our results show a shift of the density of state extrema around the Fermi level, corresponding to previously reported results,$\underline{\underline{15}}$ We also find, however, an increased density of states at the Fermi level compared to the metallic configurations for certain orientations. We find good agreement in comparison to experimentally derived density of states with the general trend of pseudogaps opening due to the bundling, $\underline{\underline{16}}$

At the end of the section we want to discuss the influence of rotation barriers on the electronic properties of monochiral achiral $\mathrm{C}_{6}$-axis bundles. At low temperatures, certain configurations of monochiral $\mathrm{C}_{6}$-axis chi- 

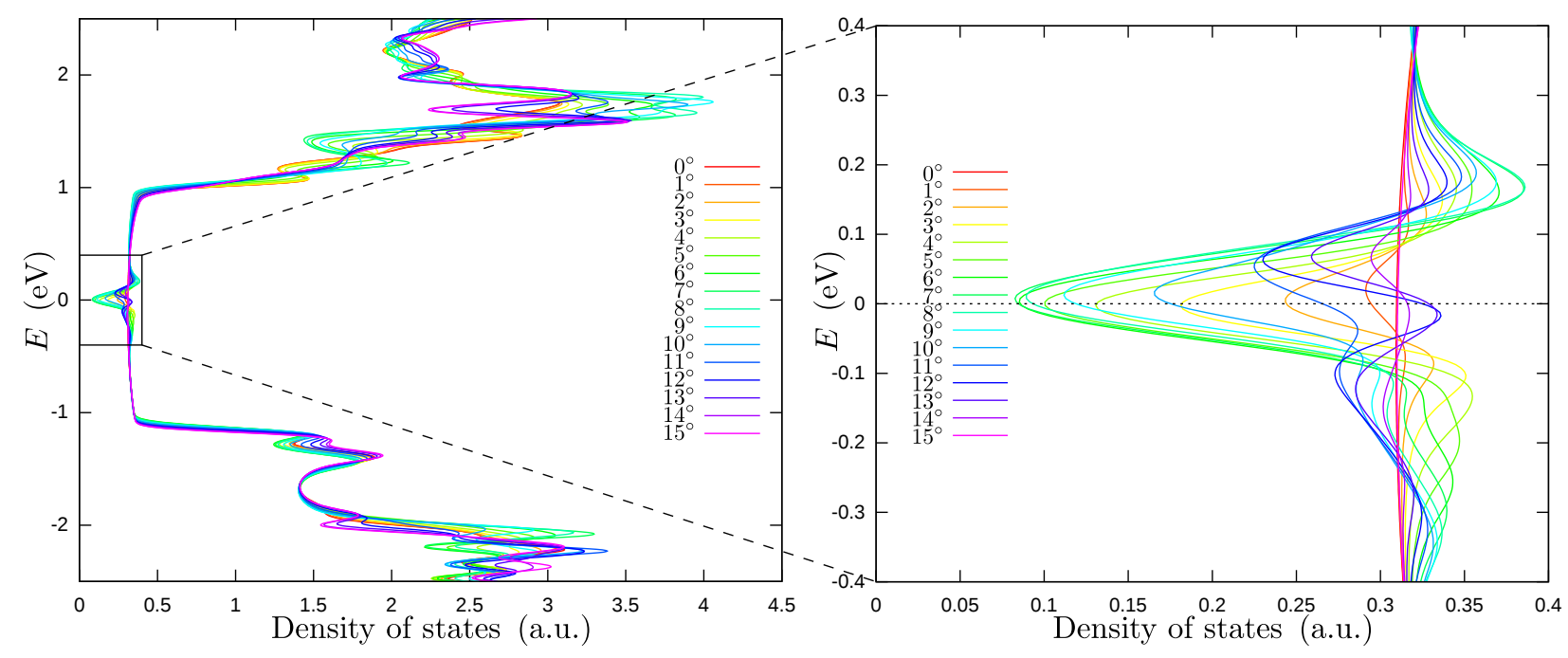

FIG. 7: Density of states of a bundle of $(6,6)$-tubes rotated starting from its high symmetry position $\left(0^{\circ}\right)$ in rainbow colors (color online). The density of states diagrams are normalized to the Fermi levels. The right panel shows the shift of the pseudogap minimum with respect to the Fermi level $(0 \mathrm{eV})$, which can also be observed in Fig. 5 .

rality bundles can be stable, e.g. the orientation at $\approx 10^{\circ}$ for the $(6,6)$-bundle. At room temperature thermal energy is likely to lead to rotations and vibrations of the tubes in the bundle. A complex electronic behavior is expected, as rotations (orientational changes) and absorption and emission occur time averaged over the experiment. Especially the slope flipping behavior and shifts of the bands lead to a further broadening of the width of the bands, which further broadens the density of states and therefore also the optical-absorption bands for the $\mathrm{C}_{6}$ axis chiralities. The high symmetry configuration with metallic behavior is the energetically most unstable configuration and therefore semi-metallic behavior has to be expected for bundles of metallic tubes. The $15^{\circ}$ configuration is a local total energy maximum and therefore also expected to be an unstable configuration, even so it is much lower in energy than the high symmetry configuration. For larger diameter tubes the rotation barriers flatten, allowing metallic behavior in $\mathrm{C}_{6}$-axis bundles of large diameter armchair tubes.

\section{CONCLUSIONS}

In summary we presented van der Waals energies and intertube distances of various chiralities of carbon nanotube bundles in dependence of the orientation of the tubes inside of the bundle. Furthermore the electronic structure of the monochiral $(6,6)$-bundle was studied in dependence of the tube orientation. For tubes with a $\mathrm{C}_{6}$ axis the orientation of the tubes in the bundle becomes a new degree of freedom to adjust the bundle properties. The intertube distance as well as the binding strength vary in dependence of the tube orientation. This dependence decreases with increasing tube diameter. Therefore this effect is mainly important for bundles composed of small diameter tubes with high curvature. By variation of the orientation of the tubes a pseudogap opens and increases until it vanishes at the next configuration which preserves the symmetry. $\mathrm{C}_{6}$-axis bundles of armchair tubes have metallic configurations. Certain configurations show a higher density of states at the Fermi level than the metallic configurations. As recent progress suggests monochiral $\mathrm{C}_{6}$-axis bundles will soon be experimentally available, which will give access to study the newly arisen bundle properties.

\section{Acknowledgments}

We acknowledge F. Hennrich, S. Heeg, A. Setaro, C. Lehmann and B. Hatting for useful discussions. This work was supported by ERC under grant no. 210642 .
* Corresponding author: heiko.dumlich@fu-berlin.de

${ }^{1}$ H. W. Kroto, J. R. Heath, S. C. O'Brien, R. F. Curl, and R. E. Smalley, Nature 318, 162 (1985).

${ }^{2}$ R. Saito, M. Fujita, G. Dresselhaus, and M. S. Dresselhaus, Phys. Rev. B 46, 1804 (1992).
${ }^{3}$ S. Iijima, and T. Ichihashi, Nature 363, 603 (1993).

4 T. W. Odom, J.-L. Huang, P. Kim, and C. M. Lieber, Nature 391, 62 (1998).

${ }^{5}$ K. S. Novoselov, A. K. Geim, S. V. Morozov, D. Jiang, Y. Zhang, S. V. Dubonos, I. V. Grigorieva, and A. A. Firsov, 
Science 306, 666 (2004).

6 R. Gillen, M. Mohr, and J. Maultzsch, Phys. Rev. B 81, 205426 (2010).

7 A. Thess, R. Lee, P. Nikolaev, H. Dai, P. Petit, J. Robert, C. Xu, Y. H. Lee, S. G. Kim, A. G. Rinzler, D. T. Colbert, G. E. Scuseria, D. Tomanek, J. E. Fischer, and R. E. Smalley, Science 273, 483 (1996).

8 M. Bockrath, D. H. Cobden, P. L. McEuen, N. G. Chopra, A. Zettl, A. Thess, and R. E. Smalley, Science 275, 1922 (1997).

9 S. Reich, C. Thomsen, and P. Ordejon Phys. Rev. B 65, 155411 (2002).

10 S. Okada, A. Oshiyama, and S. Saito, J. Phys. Soc. Jpn. 70, 2345 (2001).

11 Z. Chen and X.-Q. Wang, Phys. Rev. B 83, 081405(R) (2011).

12 J. P. Lu, Phys. Rev. Lett. 79, 1297 (1997).

13 M.-F. Yu, B. S. Files, S. Arepalli, and R. S. Ruoff, Phys. Rev. Lett. 84, 5552 (2000).

14 Y.-K. Kwon, S. Saito, and D. Tománek, Phys. Rev. B 58, R13314 (1998).

15 P. Delaney, H. J. Choi, J. Ihm, S. G. Louie, and M. L. Cohen, Phys. Rev. B 60, 7899 (1999).

16 M. Ouyang, J.-L. Huang, C. L. Cheung, and C. M. Lieber, Science 292, 702 (2001).

17 A. Szabados, L. P. Biro, and P. R. Surjan, Phys. Rev. B 73, 195404 (2006).

18 H. J. Liu, Y. W. Wen, L. Miao, and Y. Hu, Nanotechnology 18, 445708 (2007).

19 E. H. Haroz, W. D. Rice, B. Y. Lu, S. Ghosh, R. H. Hauge, R. B. Weisman, S. K. Doorn, and J. Kono, ACS Nano 4, 1955 (2010).

20 J. J. Crochet, J. D. Sau, J. G. Duque, S. K. Doorn, and M. L. Cohen, ACS Nano 5, 2611 (2011).

21 C. Blum, N. Stuerzl, F. Hennrich, S. Lebedkin, S. Heeg, H. Dumlich, S. Reich, and M. M. Kappes, ACS Nano 5, 2847 (2011).

${ }^{22}$ L. X. Benedict, N. G. Chopra, M. L Cohen, A. Zettl, S. G. Louie, and V. H. Crespi, Chemical Physics Letters 286, 490 (1998).
${ }^{23}$ L. A. Girifalco, M. Hodak, and R. S. Lee, Phys. Rev. B 62, 13104 (2000).

24 C.-H. Sun, G.-Q. Lu, and H.-M. Cheng, Phys. Rev. B 73, 195414 (2006).

25 A. I. Zhbanov, E. G. Pogorelov, and Y.-C. Chang, ACS Nano 4, 5937 (2010).

26 R. F. Rajter, R. Podgornik, V. A. Parsegian, R. H. French, and W. Y. Ching, Phys. Rev. B 76, 045417 (2007).

27 M. Seydou, Y. J. Dappe, S. Marsaudon, J.-P. Aim, X. Bouju, and A.-M. Bonnot, Phys. Rev. B 83, 045410 (2011).

28 M. Dion, H. Rydberg, E. Schröder, D. C. Langreth, and B. I. Lundqvist, Phys. Rev. Lett. 92, 246401 (2004).

29 J. Kleis, E. Schröder, and P. Hyldgaard, Phys. Rev. B 77, 205422 (2008).

30 P. Ordejon, E. Artacho, and J. M. Soler, Phys. Rev. B 53, R10441 (1996).

31 J. M. Soler, E. Artacho, J. D. Gale, A. Garcia, J. Junquera, P. Ordejon, and D. Sanchez-Portal, J. Phys.: Condens. Matter 14, 2745 (2002).

32 G. Roman-Perez, and J. M. Soler, Phys. Rev. Lett. 103, 096102 (2009).

33 J. P. Perdew, K. Burke, and M. Ernzerhof, Phys. Rev. Lett. 77, 3865 (1996).

34 N. Troullier and J. L. Martins, Phys. Rev. B 43, 1993 (1991).

35 H. J. Monkhorst, and J. D. Pack, Phys. Rev. B 13, 5188 (1976).

36 R. S. Ruoff, J. Tersoff, D. C. Lorents, S. Subramoney, and B. Chan, Nature 364, 514 (1993).

37 J. M. Cowley, P. Nikolaev, A. Thess, and R. E. Smalley, Chem. Phys. Lett. 265, 379 (1997).

38 C. S. Yannoni, R. D. Johnson, G. Meijer, D. S. Bethune, and J. R. Salem, J. Phys. Chem. 95, 9 (1991).

39 R. Tycko, R. C. Haddon, G. Dabbagh, S. H. Glarum, D. C. Douglass, and A. M. Mujsce, J. Phys. Chem. 95, 518 (1991).

40 J. P. Lu, X.-P. Li, and R. M. Martin, Phys. Rev. Lett. 68, 1551 (1992). 